\title{
Blood cells communicate remotely via an NMDA receptor-mediated
} glutamatergic chain-reaction

Olga Kopach ${ }^{* 1}$, Sergyi Sylantyev¹,2, Lucie Bard ${ }^{1}$, Piotr Michaluk ${ }^{1}$, Ana Gutierrez del Arroyo $^{3}$, Gareth L. Ackland ${ }^{3}$, Alexander V. Gourine ${ }^{4}$, Dmitri A. Rusakov ${ }^{1}$

${ }^{1}$ Queen Square Institute of Neurology, University College London, Queen Square, London WC1N 3BG, UK

${ }^{2}$ Rowett Institute, University of Aberdeen, Ashgrove Rd. West, Aberdeen AB25 2ZD, UK

${ }^{3}$ Translational Medicine and Therapeutics, Queen Mary University of London, Mile End Road, London E1 4NS, UK

${ }^{4}$ Department of Neuroscience, Physiology and Pharmacology, University College London, Gower Street, London WC1E 6BT, UK

* Equal contribution

Correspondence: d.rusakov@ucl.ac.uk; o.kopach@ucl.ac.uk; s.sylantyev@abdn.ac.uk 


\begin{abstract}
Neutrophils are white blood cells that are critical to the acute inflammatory and adaptive immune responses. While their diverse interactions with tissue cells and pathogens have been intensely studied, little is known about how they communicate among themselves. Here we employ up-to-date neuroscience techniques to find that electrical, mechanical, or ligand stimulation of an individual neutrophil triggers $\mathrm{Ca}^{2+}$ mobilisation in nearby cells by engaging their NMDA receptors. Glutamate imaging reveals that the activated cells in turn release glutamate thus triggering a chain reaction that generates long-range signal exchange among the neutrophil assembly. These observations uncover a regenerative-wave mechanism - NMDA receptor dependent glutamateinduced glutamate release - that enables remote communication, or a signal-spreading 'detonation' - among blood cells.
\end{abstract}




\section{Introduction}

Neutrophils, a type of white blood cells, provide the first line of defence against pathogens during acute inflammation (1-4). In humans, a significant decrease in neutrophil numbers could lead to severe immunodeficiency or death. These polymorphonuclear leukocytes mobilise to the site of inflammation and engage several cell-killing mechanisms to clear the infection $(5,6)$. During this process, pathogens trigger a multi-stage vascular-to-tissue neutrophil recruitment cascade: freely circulating cells start rolling, adhering, and crawling along the vascular walls, followed by transmigration out of the blood vessel $(7,8)$. In addition to targeting localised inflammation, neutrophils are involved in the systemic body response to an insult. Notably, they contribute to autoimmune reactions, such as rheumatoid arthritis, and to allergic reactions, such as anaphylaxis, in which antibodies recognize either self- or foreign antigens (9). Blood neutrophils can thus act as immediate effectors of immune reactions, mediating a pathological development, such as inflammation-associated cancer metastasis $(10,11)$. Evidence has been emerging for intense communication of neutrophils with other actors of pathological immune response, such as platelets, Tcells, or infectious agents (12-14). However, through which mechanisms neutrophils could communicate, among themselves and with other freely-moving cells, remain poorly understood.

$\mathrm{Ca}^{2+}$ signalling has long been considered key to the physiological functions of neutrophils (15), which are equipped with a variety of cell-surface receptors, including GPCRs, FcRs, and integrins, capable of mediating $\mathrm{Ca}^{2+}$ messages (16-18). The activation mechanism often involves adhesion effectors and a cooperation between store-operated calcium entry (SOCE) and plasma-membrane calcium-release activated $\mathrm{Ca}^{2+}$ (CRAC) channels (19). Human neutrophils can release glutamate (20), in response to either the protein kinase $\mathrm{C}$ activation or NMDA receptor (NMDAR) ligands (21). The blood level of glutamate could be an important indicator of immune response, in particularly after trauma (22), during which neutrophil hyperactivity can lead to tissue damage (23). However, what mechanism can prompt neutrophils to release glutamate en masse remains unknown.

Whether neutrophils respond to a synchronously released effector or whether they communicate among themselves to produce a co-ordinated mass action is therefore an important and intriguing question. In a wider context, the question is whether neutrophils, or other blood cells for that reason, can act as a self-contained signalling network rather than independent cellular entities. Networking is a universal trait of brain cells, which prompted us to employ advanced neuroscience techniques in an attempt to understand whether human neutrophils can communicate among themselves as a cell ensemble. The results unveil a previously unrecognised chain-reaction mechanism that can generate self-maintained, regenerative waves of cellular physiological action across the population of spatially separated neutrophils. 


\section{RESULTS}

\section{NMDARs contribute to $\mathrm{Ca}^{2+}$ signalling in human neutrophils}

First, we sought to probe directly the function of NMDARs that have been associated with glutamate release by neutrophils (21). We held individual neutrophils in whole-cell mode (current clamp; zero- $\mathrm{Mg}^{2+}$ solution): while the resting membrane potential of neutrophils is near $-75 \mathrm{mV}$ (24), it rapidly depolarised upon break-in (Fig. S1A). We next applied NMDA $(100 \mu \mathrm{M})$ and the co-agonist glycine $(1 \mathrm{mM}, 1 \mathrm{~s}$ square pulse) using a rapid-solution-exchange system (Fig. 1A) (25). The application stimulus evoked a clear inward current, which was blocked either by extracellular $\mathrm{Mg}^{2+}(2 \mathrm{mM})$, by the NMDAR antagonist APV $(50 \mu \mathrm{M})$, or by the selective antagonist of NR2B-containing NMDARs Co101244 (1 $\mu \mathrm{M}$, Fig. 1B-C). Consistent with strong $\mathrm{Ca}^{2+}$ permeability of activated NMDARs, $\mathrm{Ca}^{2+}$ imaging (with Fluo-4 AM) revealed that bath application of NMDA (100 $\mu \mathrm{M})$ and glycine $(50 \mu \mathrm{M})$ triggered a rise in intracellular $\mathrm{Ca}^{2+}$ (Fig. 1D), which was blocked by extracellular $\mathrm{Mg}^{2+}(2 \mathrm{mM})$ or by Co101244 (Fig. 1E-F). Notably, with or without NMDAR blockade, a subsequent application of the protein kinase $C$ activator phorbol myristate acetate (PMA, $1 \mu \mathrm{M}$ ) triggered a prominent $\mathrm{Ca}^{2+}$ elevation (Fig. 1E-F). We found that cell depolarisation (a series of input current square pulses, whole-cell current mode) also produced a robust $\left[\mathrm{Ca}^{2+}\right]_{\text {in }}$ rise, which was insensitive to APV (Fig. $1 \mathrm{H})$. These observations pointed to multiple mechanisms of $\mathrm{Ca}^{2+}$ mobilisation in neutrophils, with NMDARs providing a major pathway.

\section{One neutrophil can trigger multi-cell $\mathrm{Ca}^{2+}$ response}

PMA-induced $\mathrm{Ca}^{2+}$ mobilisation in neutrophils has earlier been shown to induce glutamate release (21) whereas our data relate both PMA and glutamate receptors to a rise in $\left[\mathrm{Ca}^{2+}\right]_{\text {in }}$ (Fig. 1). Could such mechanisms thus enable remote glutamatergic signalling among neutrophils? Our initial experiments indicated that depolarisation of one neutrophil could lead to moderate $\left[\mathrm{Ca}^{2+}\right]$ in elevations in neighbouring cells (Fig. S1B; Supplementary Video 1 FigS1B). To rule out any optical concomitants of such effects (such as light scattering), we sought to obtain direct electrophysiological evidence for neutrophil-neutrophil interaction. We held two neighbouring cells (5-15 $\mu \mathrm{m}$ apart) in whole-cell mode (Fig. 2A). Remarkably, applying a depolarising stimulus to one cell induced an inward current in the other (Fig. 2A-B). The response was blocked by APV (Fig. 2B) and occurred 70-140 ms after the stimulus (Fig. 2C). This delay is in line with the diffusion time of glutamate molecules (diffusioncoefficient in ACSF D $=0.86$ $\mu \mathrm{m}^{2} / \mathrm{ms}$ ) that should cover such distances in $20-40$ ms before rising to a concentration sufficient to activate NMDARs. 
A

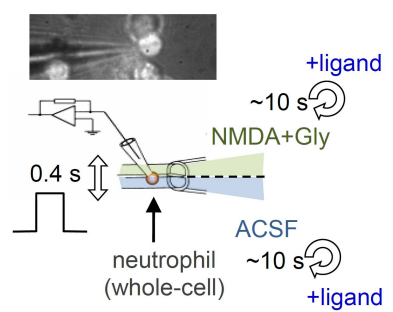

B
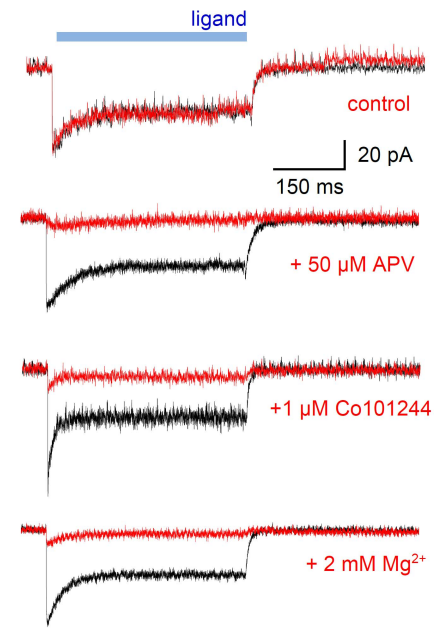

C

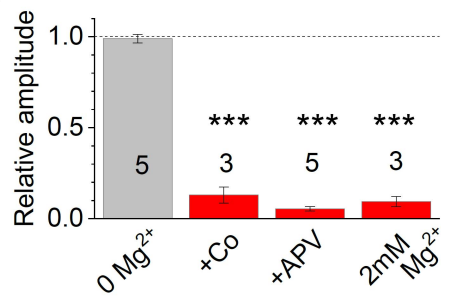

D $\quad \mathrm{Mg}^{2+}$

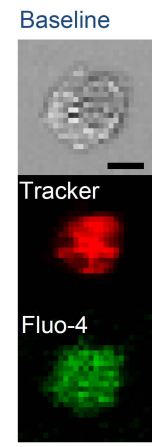

$0-1$

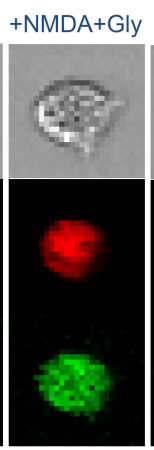

2-3

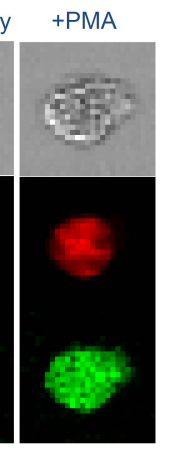

$5-6 \mathrm{~min}$
E

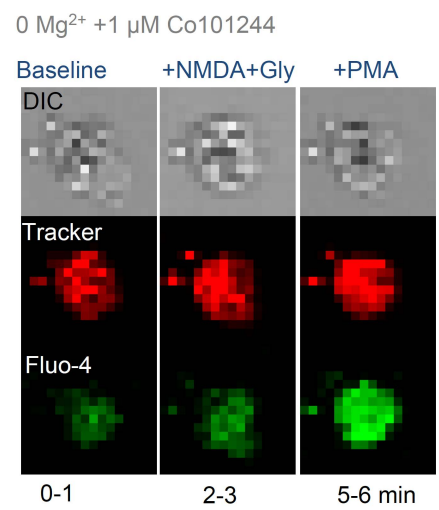

$\mathrm{F}$

G

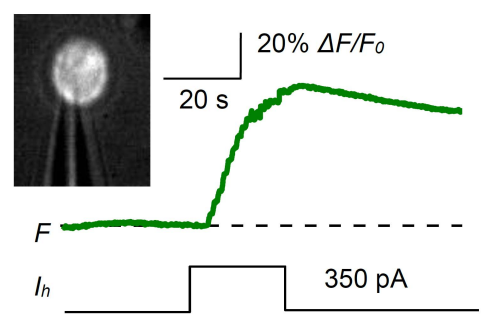

$\mathrm{H}$

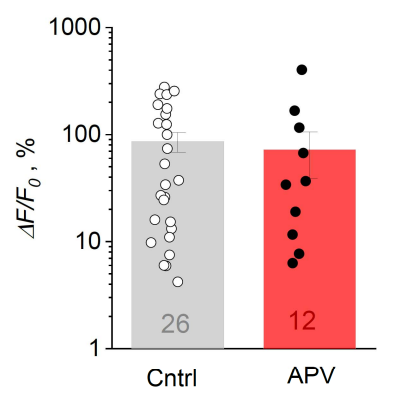

Figure 1. Activation of NR2B-containing NMDA receptors in human neutrophils.

(A) Diagram depicting probing of NMDARs with a rapid-exchange system (25): a neutrophil (held in wholecell) is stimulated pharmacologically by applying different solutions through two channels of $\theta$ glass pipette (tip diameter $\sim 200 \mu \mathrm{m}$ ) mounted on an electronically controlled piezo-drive to enable the ultra-fast delivery $(<1 \mathrm{~ms}$ resolution; solutions in each channel exchanged within $10 \mathrm{~s})$.

(B) Representative wholecell currents recorded in human neutrophils in response to locally applied NMDA ( $1 \mathrm{mM}$ ) and glycine (1 mM), in zero $\mathrm{Mg}^{2+}, 2 \mathrm{mM}$ $\mathrm{Mg}^{2+}$, and in the presence of NMDAR antagonists APV $(50 \mu \mathrm{M})$ and $\mathrm{Co101244}(1$ $\mu \mathrm{M})$ in zero $\mathrm{Mg}^{2+}$ (control), as indicated; one-cell pharmacological manipulations applied at a $\sim 20$ s interval.

(C) Summary of experiments shown in (B); mean \pm SEM (amplitude over 300-500 ms of the pulse), normalised to control (sample size shown). ${ }^{* * *} p<0.01$.

(D) Characteristic images of a neutrophil (grey DIC image, top raw) loaded with Cell tracker (red channel, middle) and Fluo 4-AM (green, bottom), before and after bath application (2-3min duration) of NMDA (100 $\mu \mathrm{M})$ and glycine $(50 \mu \mathrm{M})$, and added PMA $(1 \mu \mathrm{M})$, as indicated; experimental timing as shown; scale bar,

$5 \mu \mathrm{m}$.

(E) Experiment as in (D), but in the presence of the selective NR2B-containing NMDAR antagonist Co101244 (1 $\mu \mathrm{M})$.

(F) Statistical summary of experiments shown in (D-E): $\Delta G / R$, $\mathrm{Ca}^{2+}$ sensitive fluorescent green Fluo-4 signal $(\Delta G)$ normalised to the red signal $(R)$ to account for fluctuations in focus / brightness; bars, mean \pm 
SEM normalized to control (sample size shown); ${ }^{* * *} p<0.001$ (one-way ANOVA, Bonferroni post-hoc test).

(G) One-cell example showing that membrane depolarization current induces $\mathrm{Ca}^{2+}$ mobilization in the neutrophil (held in whole-cell); inset, DIC+Fluo-4 AM image; traces: F, Fluo-4 fluorescence signal; Ih, holding current.

$(\mathrm{H})$ Summary of experiments shown in $(\mathrm{G})$ : depolarization-induced $\left[\mathrm{Ca}^{2+}\right]$ in rise represented by the $\Delta F / F_{0}$ Fluo-4 signal; dots, individual cells; bars: mean \pm SEM; sample size shown.

A

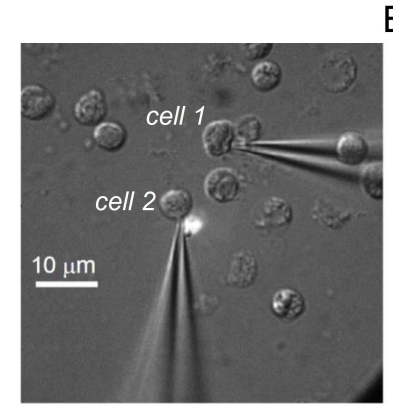

B

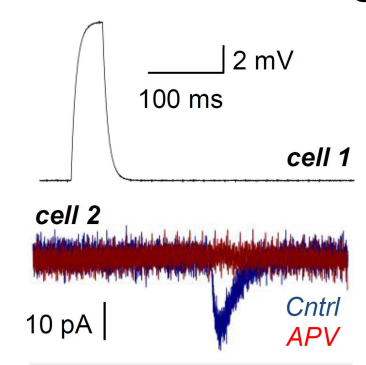

D
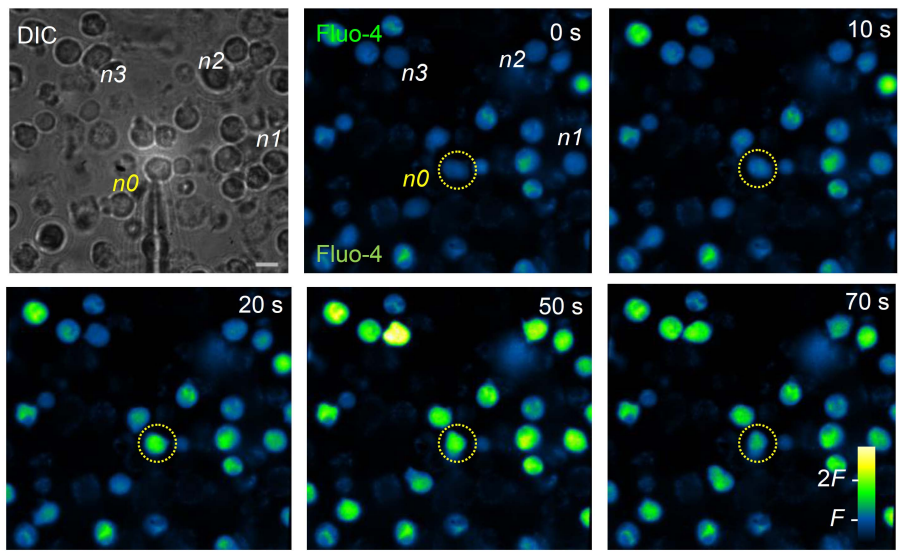

E
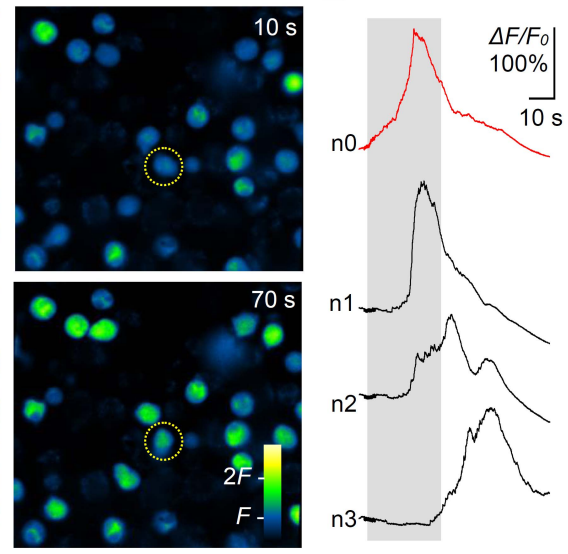
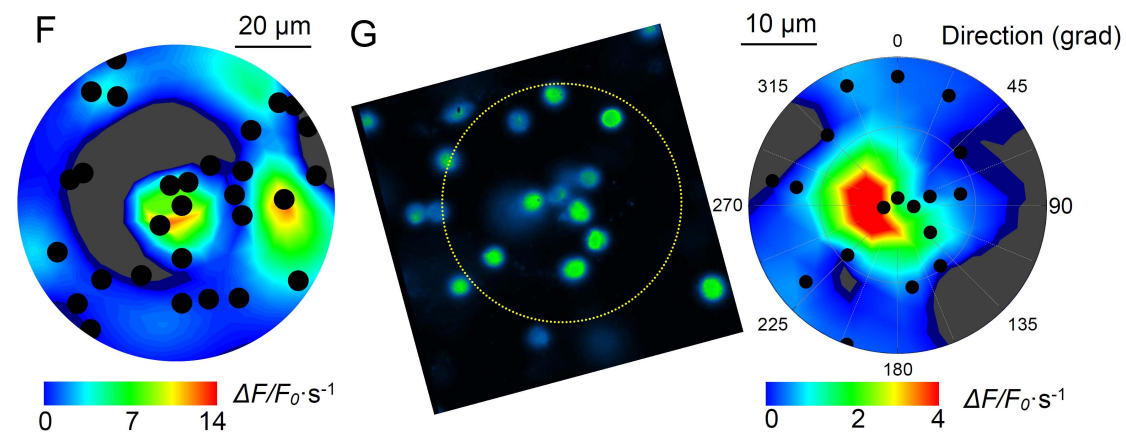

enabling one-cell electrical stimulation; colour panels, time-lapse images (Fluo-4 channel); electric stimulus (50 pA current) applied to the patched cell during 0-40 s period; false colour scale, $\mathrm{Ca}^{2+}$ dependent fluorescence intensity $(F)$; see Supplementary Video 2 Fig2D. 
(E) Examples of fluorescence signal recordings from cells $n 0-n 2$ in (D), as indicated; grey shadow, current stimulus in cell $n 0$.

(F) Diagram mapping the stimulus-induced $\mathrm{Ca}^{2+}$ wave propagating across scattered neutrophils (black circles): map of the maximum rate of $\left[\mathrm{Ca}^{2+}\right]$ in rise in individual cells (color-coded; stimulated neutrophil at the centre) over the recording time; separate wave-fronts can be seen.

(G) Another example, in conditions similar to $(F)$, showing distinct fronts of propagating waves of $\left[\mathrm{Ca}^{2+}\right]$ in rise across the cell ensemble.

Although these experiments had an exceptionally low success rate due to the high mobility of neutrophils (5 out of 57 successful one-cell patches), they provide direct evidence for remote 'excitatory' signalling between neutrophils. To understand whether and how such signalling leads to mobilisation of intracellular $\mathrm{Ca}^{2+}$, we monitored intracellular $\mathrm{Ca}^{2+}$ in neutrophil populations, across the region of interest, using timelapse (wide-field) fluorescence $\mathrm{Ca}^{2+}$ imaging. We found that a depolarisation stimulus applied to one patched cell, triggered a wave of $\mathrm{Ca}^{2+}$ rises in a significant proportion (20-50\%) of neutrophils, which propagated gradually towards distal cells (Fig. 2D-E; Supplementary Video 2 Fig2D). The effect was observed consistently, across different cell preparations ( $n=201$ cells in 25 independent experiments). Intriguingly, the magnitude of $\mathrm{Ca}^{2+}$ signals was not decaying with the distance from the stimulated cell, but rather showed a self-maintained propagating wave front (Fig. 2F-G, Supplementary Video 3 Fig2G).

\section{Signal exchange among neutrophils is predominantly NMDAR-dependent}

To probe receptor mechanisms of this phenomenon in an unbiased manner, we devised an experiment in which one-half of the neutrophil population was exposed to the control extracellular medium whereas the other half received a medium containing receptor blockers (Fig. 3A). We thus found that the single-cell stimulus-induced $\mathrm{Ca}^{2+}$ wave in neutrophils was significantly inhibited in the presence of APV (by $\sim 60 \%, \mathrm{n}=46, p<$ 0.001; Fig. 3B; Supplementary Video 4 Fig3AB). The effect was similar without subdividing the medium (inhibition by $40.5 \%, \mathrm{n}=109$ cells pooled from 15 independent experiments, $p=0.0012)$, and with another NMDAR antagonist, CPP ( $10 \mu \mathrm{M}$; reduction of $\sim 82 \% ; n=10, p=0.0322$ ). Interestingly, APV did not suppress $\mathrm{Ca}^{2+}$ mobilisation in the stimulated cell: in contrast, adding the non-specific metabotropic glutamate receptor antagonist MCPG $(100 \mu \mathrm{M})$ blocked $\mathrm{Ca}^{2+}$ rises in the stimulated neutrophil while having little effect on $\mathrm{Ca}^{2+}$ signalling in remote cells (Fig. 3C).

We noticed that in these experiments, the stimulated cell triggered mass $\mathrm{Ca}^{2+}$ mobilisation not only upon current injection but also in response to a mechanical touch by the patch-pipette (Fig S2; Supplementary Video 5 FigS2A). Stimulated neutrophils also showed remarkable morphological motility, which was generally coincident with $\left[\mathrm{Ca}^{2+}\right]_{\text {in }}$ rises, by rapidly extending their filopodia, Fig. S3, Supplementary Video 6 FigS3), consistent with previous reports (26). Our subsequent tests confirmed that 
mechanical stimulation of one cell alone was indeed capable of producing the effect similar to that induced by current injection (Fig. 3D-F; Supplementary Video 7 Fig3D).

Taken together, these observations suggest that activation of one neutrophil, either electrical or mechanical, could induce a spreading wave of $\mathrm{Ca}^{2+}$ mobilisation in the local neutrophil population. However, to generate a regenerative wave of activity spreading in space, one has to have a distributed mechanism that 'reproduces' the original stimulus. Because neutrophils are activated by glutamate (Fig. 1) and can release glutamate (20, 21 ), we asked whether glutamate-induced glutamate release is indeed the mechanism in question.

A

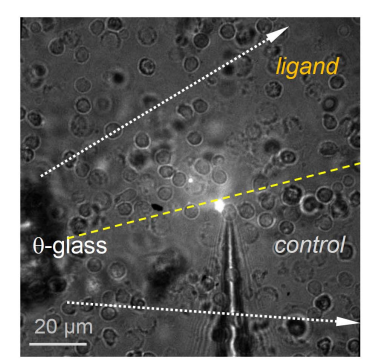

C

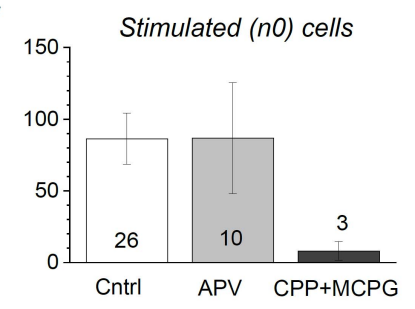

D
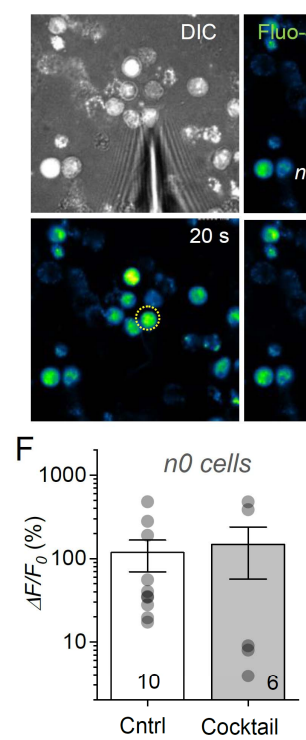

B
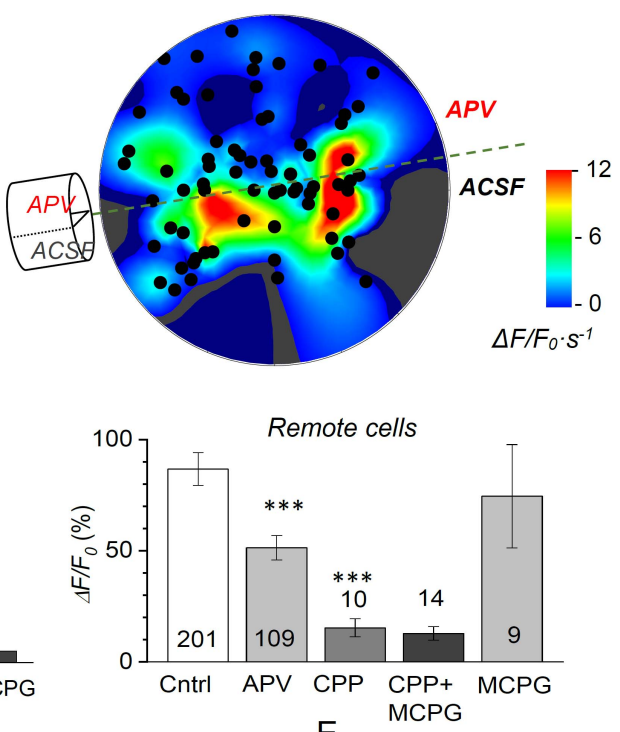

E
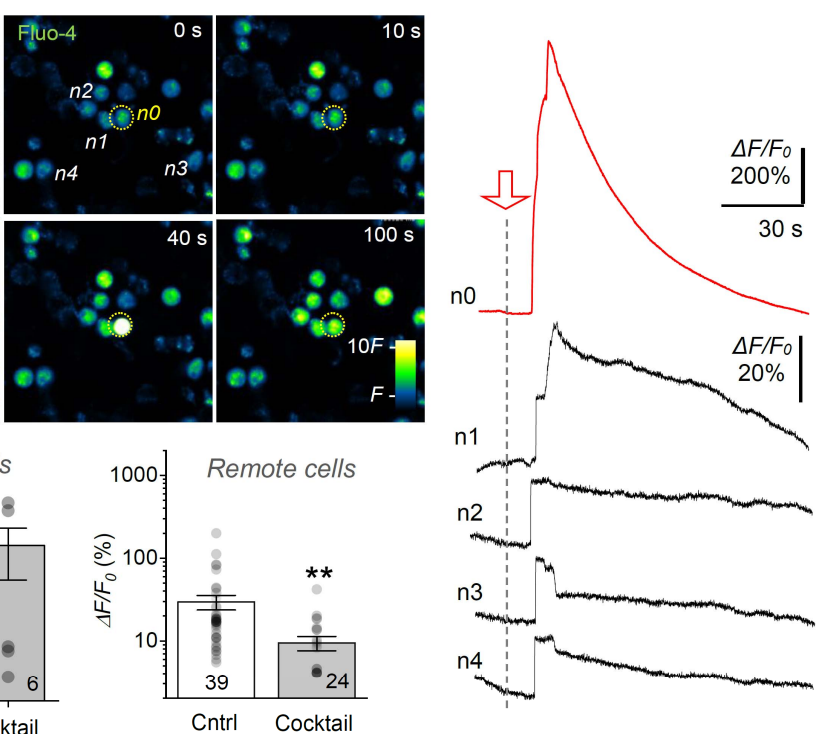

Figure 3. Electrical or mechanical stimulation of one neutrophil induces a self-propagating wave of glutamate receptordependent $\mathrm{Ca}^{2+}$ rise in the cell scatter.

(A) Experiment in which one half of the neutrophil suspension was exposed to the control bath medium whereas the other half received $50 \mu \mathrm{M}$ APV, as indicated, using thetaglass application; a patch pipette is seen.

(B) Mapping the maximum rate of $\left[\mathrm{Ca}^{2+}\right]$ in rise in individual cells (dark circles; signal color-coded as indicated) following electric stimulation of one neutrophil (bright cell in A); current injection triggered a spreading wave of $\mathrm{Ca}^{2+}$ rises across the control half but less so in the APV-containing half; see Supplementary Video 4 Fig3AB.

(C) Summary of experiments shown in (A), for control conditions and in the presence of glutamate receptor antagonists APV (100 $\mu \mathrm{M}), \mathrm{CPP}(10 \mu \mathrm{M})$, and MCPG $(400 \mu \mathrm{M})$. Bars, mean \pm SEM; ${ }^{* *} p<0.01$ (unpaired t-test; sample size shown).

(D) Grey image, a suspension of Fluo-4 AM loaded human neutrophils (DIC channel); cell $n 0$ is mechanically stimulated (lightly tapped by a patch pipette) for $\sim 2 \mathrm{~s}$, onset at $8 \mathrm{~s}$, which is followed by $20 \mathrm{~s}$ current injection (onset 
at $22 \mathrm{~s}$ ). Colour panels, time-lapse recording (Fluo-4 channel); false colour scale, $\mathrm{Ca}^{2+}$-dependent fluorescence intensity $(F)$, as indicated; see Supplementary Video 7 Fig3D.

(E) Examples of fluorescence signal recordings $\left(\Delta F / F_{0}\right)$ in cells $n 0-n 4$ shown in $(D)$ after mechanical (red arrow) stimulation of cell $n 0$.

(F) Summary of experiments shown in (D-E); bars, mean \pm SEM; dots, individual data points; ${ }^{* *} p<0.01$ (unpaired t-test; sample size shown); cocktail of antagonists: APV (100 $\mu \mathrm{M}), \mathrm{CPP}(10 \mu \mathrm{M})$, and MCPG $(400 \mu \mathrm{M})$.

\section{Glutamate-induced glutamate release underpins NMDAR-dependent 'chain reaction' among neutrophils}

First, we used a glutamate-specific biosensor (27) (Fig. S3C) to find that electric stimulation (current injection) of an individual neutrophil produces a prominent glutamate response (Fig. S3D), which was in line with earlier observations $(20,21)$. However, the sensor reports glutamate responses that are heavily filtered in space and time whereas a regenerative (wave-like) signalling mechanism must involve heterogenous spatiotemporal landscapes of the glutamate.

To enable glutamate detection across the field of view, we employed an enzymaticbased assay (28-30). This approach relies on the glutamate-dependent NADH fluorescence in the enzyme-containing medium (Fig. 4A), showing prominent fluorescence responses to brief (100 ms) local glutamate puffs (Fig. 4C). In the initial high-resolution experiments, we combined this method with time-lapse imaging to detect pronounced glutamate hotspots that were generated, upon mechanical stimulation of one neutrophil, both in stimulated and in the neighbouring cell (Fig. S4). We next expanded these tests to a larger field of view. Again, stimulation of one cell showed prominent glutamate responses both in the stimulated cell, and in neutrophils that were tens of microns away (Fig. 4D-E, Supplementary Video 8 Fig4D).

Remarkably, individual remote neutrophils showed motile, oscillating hotspots of glutamate, presumably reflecting changes in its release sites in the cell membrane (Fig. S4B).

Comparing these data to the glutamate-calibrated NADH signal indicated that the endogenously released glutamate could reach a hotspot level of $5 \square \mathrm{M}$, both near the stimulated cells $(n=17)$ and remote neutrophils $(n=78 ; p=0.362$ compared with $5 \square$ M exogenous glutamate; Fig. 4F). A similar level of glutamate was reported earlier for neutrophils activated with PMA (21), which appears close to the $\sim 8 \square$ M concentration considered optimal for neutrophil chemotaxis and cytoskeleton polarization (31). This 'chain reaction' of glutamate release was inhibited by the NMDAR antagonist CPP: its blocking effect in remote cells was $\sim 69 \%$ ( $n=29$, $p<0.001$; Fig. $4 \mathrm{G}$ ) and $\sim 81 \%$ when applied together with the mGluR antagonist MCPG ( $n=59, p<0.001$; Fig. 4G, Supplementary Video 9 Fig4DAPV). The glutamate receptor antagonist cocktail had little effect on the glutamate release by the stimulated neutrophil $(n=17$ in control and $n$ $=4$ in CPP, $p=0.383$, and $n=11$ in CPP with MCPG, $p=0.102$; Fig. 4G;

Supplementary Video 9 Fig4DAPV), suggesting that an independent mechano-sensing mechanism (likely associated with the TRP receptor family) was involved. 
A

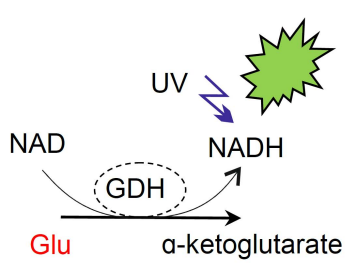

B

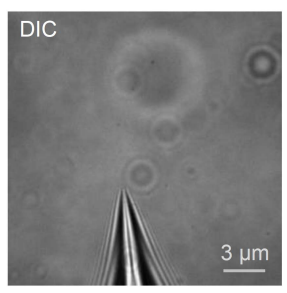

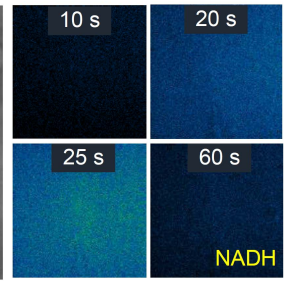

C

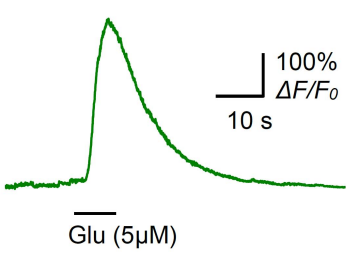

E
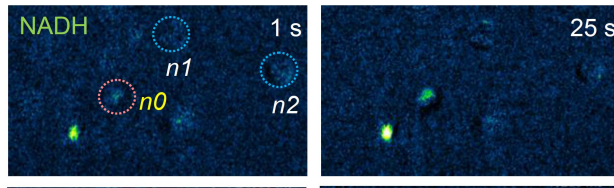

$100 \mathrm{~s}$
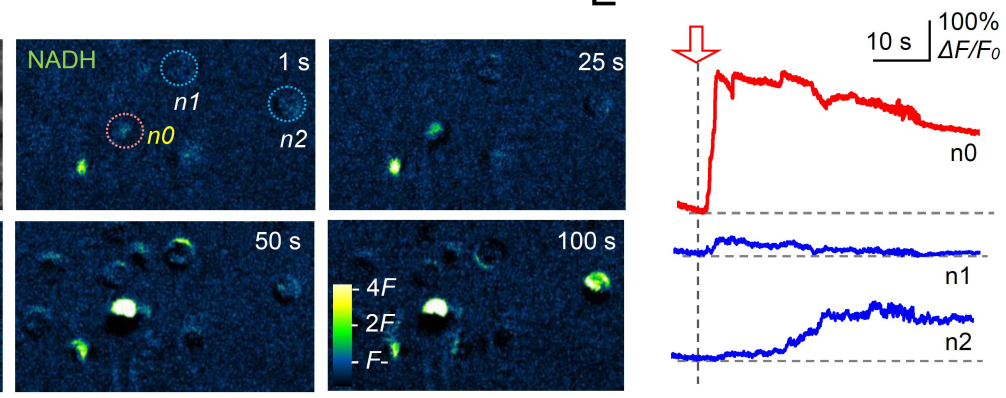

$\mathrm{F}$

G

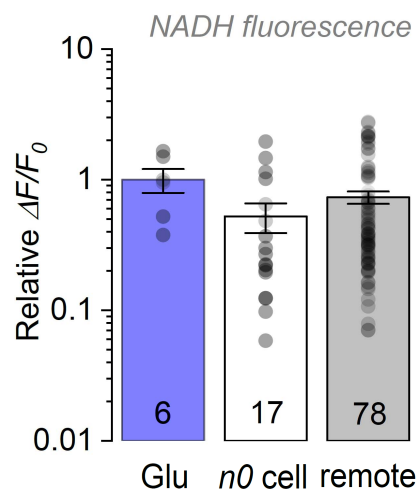

Remote
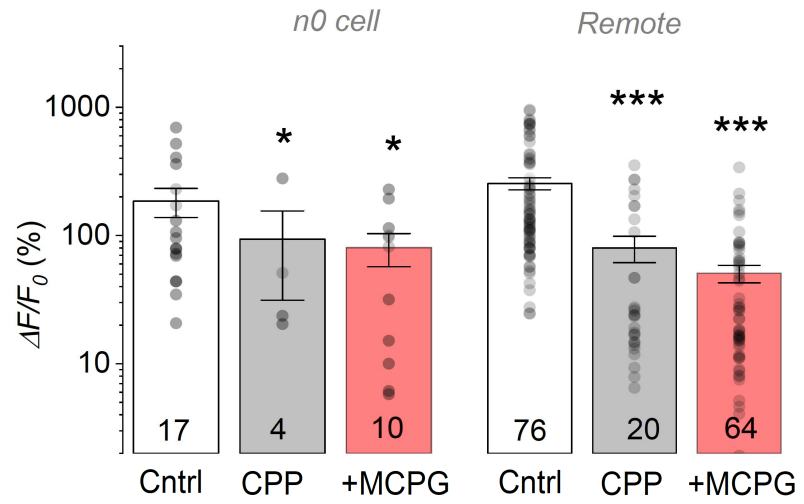

Figure 4. Glutamate imaging reveals glutamate-induced glutamate release prompting a positive-feedback reaction in a scatter of human neutrophils.

(A) Diagram, fluorescence imaging an enzymatic-based assay: conversion of $\beta$-nicotinamide adenine dinucleotide (NAD) by L-glutamic dehydrogenase $(G D H)$ (in the presence of glutamate) to NADH that fluoresces upon UV light excitation.

(B) Glutamate imaging, method validation: DIC image, patch-pipette puffing glutamate $(5 \mu \mathrm{M}, 10 \mathrm{~s})$ into the enzymatic-based medium containing $\mathrm{GDH}(60 \mathrm{U} / \mathrm{ml})$ and NAD $(1 \mathrm{mM})$; colour panels: examples of time-lapse snapshots (time stamp shown) of NADH fluorescence.

(C) Time course of NADH-mediated fluorescence in the experiment shown in (B).

(D) DIC image, human neutrophils bathed in enzymatic medium, with cell $n 0$ stimulated mechanically (patch pipette shown); colour panels (NADH channel), time-lapse snapshots following mechanical stimulation $n O$ cell (onset $\sim 2 \mathrm{~s}$, time stamps shown); false colour scale, $\mathrm{NADH}$-dependent fluorescence intensity $F$ (image in basal conditions before $t=0$ was subtracted throughout); see Supplementary Video 8 Fig4D.

(E) Time course of NADH fluorescence in three selected cells (n0-2), as in (D); red arrow, mechanical stimulus onset.

(F) Summary of tests shown in (B-E): relative $\Delta F / F_{0}$ signals produced by a $5 \mu M$ glutamate puff (set as $100 \%$ ), and in the experiments shown in (D-E), as indicated; bars: mean \pm SEM (sample size shown). 
(G) Experiments shown in (D-E) for stimulated ( $n 0$, left) and scattered (right) cells; in control conditions and after application of the NMDAR antagonist CPP $(10 \mu \mathrm{M})$, or an antagonist cocktail $(100 \mu \mathrm{M}$ APV, $10 \mu \mathrm{M}$ CPP, $400 \mu \mathrm{M}$ MCPG); bars: mean \pm SEM (dots, individual data points; sample size shown). *p < $0.05,{ }^{* * *} p<0.001$ (unpaired t-test).

\section{Localised glutamate pulse reproduces the effect of neutrophil stimulation}

Finally, we asked if a local glutamate hotspot on its own would exert a similar chainreaction effect in a cell population. First, we confirmed that a rapid application of glutamate using a theta-glass system (Fig. 1A) induced in neutrophils a characteristic inward current, which could be partly inhibited by the NMDAR antagonist APV, and fully suppressed by the further addition of pertussis toxin (inhibitor of G-protein activity; Supplementary Fig. S5). We next found that a $100 \mathrm{~ms}$ local pulse of glutamate $(10 \mu \mathrm{M})$ with glycine (100 $\square \mathrm{M}$; Supplementary Video 10 Fig5B) evoked a robust $\left[\mathrm{Ca}^{2+}\right]_{\text {in }}$ rise in the neutrophils adjacent to the pipette tip ( $n=7$; Fig. 5A-B), followed by a wave of $\left[\mathrm{Ca}^{2+}\right]_{\text {in }}$ rises in remote neutrophils (Fig. 5C-D; Supplementary Video 11 Fig5C). The glutamate-induced $\left[\mathrm{Ca}^{2+}\right]_{\text {in }}$ rise was dramatically reduced in the absence of glycine, both in the pipette-adjacent (drop in the Fluo-4 ratio from $\sim 61.6 \%, \mathrm{n}=7$ to $\sim 4.7 \%, \mathrm{n}=4$ ) and distal neutrophils (from $\sim 56.1 \%, \mathrm{n}=66$ pooled from 14 independent experiments, to $\sim 12.8 \%, n=9, p<0.05$; Fig. 5E). These results lend further support to the key role of glutamatergic, NMDAR-dependent signalling as the mechanism underpinning remote communication among neutrophils.
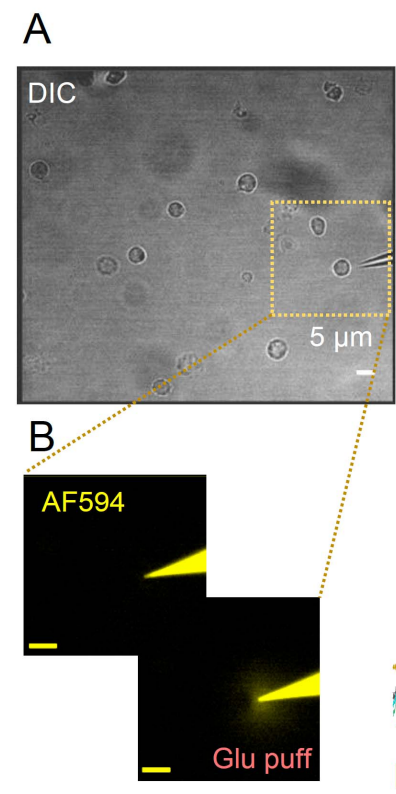

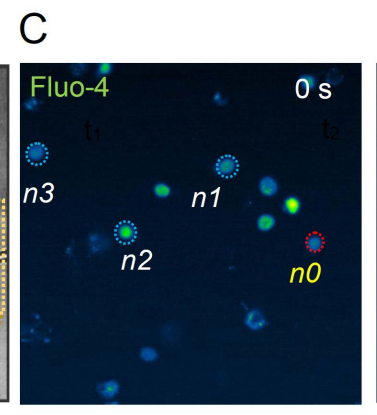

$\mathrm{D}$

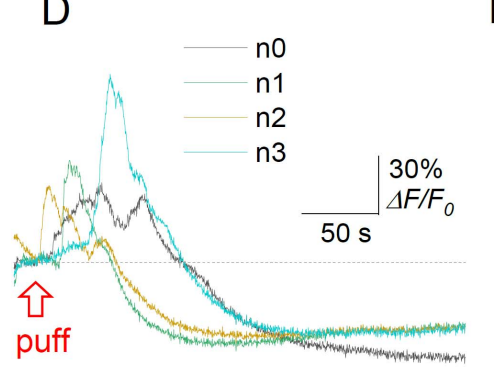

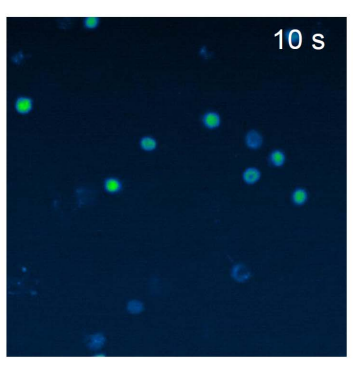

$\mathrm{E}$
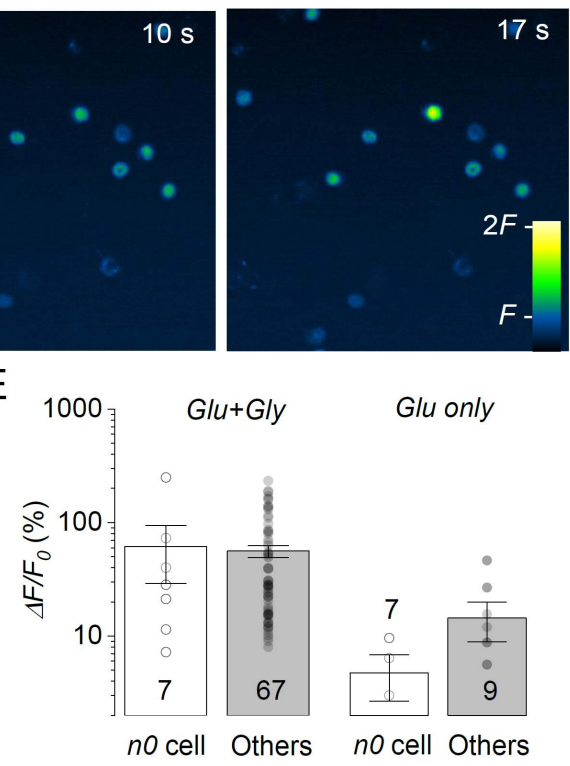

Glu only

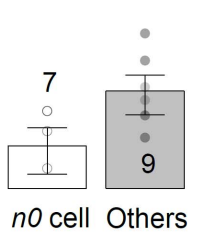

Figure 5. Brief local application of glutamate triggers a wave of $\mathrm{Ca}^{2+}$ elevations across neutrophil population.

(A) DIC image depicting a pressurised micropipette for rapid glutamate application in a group of neutrophils. 
(B) Two snapshots of the micropipette tip $(10 \mu \mathrm{M}$ glutamate, $100 \mu \mathrm{M}$ glycine, $200 \mu \mathrm{M}$ fluorescence tracer Alexa Fluor 594, in bath medium), in resting conditions and during glutamate puff, as indicated; scale bar as in A; see Supplementary Video 10 Fig5B for full puff recordings.

(C) Snapshots from time-series fluorescence imaging (Fluo-4 channel, field of view as in A) depicting $\mathrm{Ca}^{2+}$ rises upon a 100 ms glutamate puff (bear cell $n 0$, onset $9 \mathrm{~s}$ ); false colour scale; see Supplementary Video 11 Fig5C.

(D) Fluo-4 $\Delta F / F_{0}$ signal traces in the four cells depicted in $\mathrm{C}$, as indicated.

(E) Statistical summary of experiments shown in A-C; average maximum $\Delta F / F_{0}$ Fluo-4 signal elevation (bars, mean \pm SEM; dots, individual data points; sample sizes shown) for the nearest neutrophil to the puff ( $n 0$ cell) and other cells in the field of view.

\section{DISCUSSION}

Here, we have found that human neutrophils, blood cells at the first line of defence against body pathogens, are capable of communicating remotely, by releasing and sensing the excitatory neurotransmitter glutamate, in an NMDA receptor dependent manner. Because in response to glutamate individual cells boost their own glutamate release, this positive feedback mechanism appears to generate a self-sustained (chainreaction type) signalling wave that can propagate through the neutrophil assembly. This mode of regenerative intercellular communication unveils a cooperative action of neutrophils, possibly arising as a communal reaction to what could be a pathogenic stimulus sensed by just one cell. One possibility is that the glutamate-dependent glutamate release by neutrophils helps control glutamate concentration in the bloodstream, which is a key aspect of body's immune response (22). Indeed, varying glutamate level and exposure has a prominent effect on normal and autoimmune human T-cells $(32,33)$, which also plays a key role in what has been considered as a neuroimmune network (34-36). Whether the glutamate-induced glutamate release wave by neutrophils thus provides a 'detonator' signal to other immune cells, as a response to a local stimulus, remains an important and in intriguing question.

The lifespan and physiological destiny of a neutrophil includes several highly distinct stages. The present findings should be considered relevant to the cells 'free-circulating' in the blood stream, before they migrate out of the blood vessel. Revealing the signalling mode(s) among human neutrophils that crawl along vascular walls and transmigrate into the tissue $(7,8)$ will require experimental settings that provide a realistic representation of such conditions in situ. 


\section{METHODS}

Preparation of human neutrophils. Neutrophils were isolated from blood samples obtained by venepuncture from healthy volunteers according to protocols approved by UCL Research Ethics Committee (University College London Institute of Neurology, London, UK), with the informed consent of participants.

For isolation of neutrophils, we used a method of dextran sedimentation and differential centrifugation through a Ficoll-Hypaque density gradient as described in detail elsewhere (37). Briefly, a sample of the whole blood was suspended in sodium citrate solution, and a suspension of neutrophils was obtained by sedimentation in $2 \%$ dextran (Sigma, UK) in $0.9 \% \mathrm{NaCl}$ for $45-60 \mathrm{~min}$ at room temperature. The neutrophil-enriched upper layers of the suspension were collected and centrifuged (1150 rpm, $10 \mathrm{~min}$ at 4 $\left.{ }^{\circ} \mathrm{C}\right)$. Residual erythrocytes were removed by hypotonic lysis, and the obtained suspension was further centrifuged (1300 rpm, 6 min at $4{ }^{\circ} \mathrm{C}$ ); the pellet was further resuspended in PBS and purified by gradient centrifugation over Ficoll-Hypaque (Sigma, cat. 1077; $1500 \mathrm{rpm}, 30 \mathrm{~min}$ at $4^{\circ} \mathrm{C}$ ). The resulting pellet containing neutrophils was finally re-suspended in $\mathrm{HBSS}\left(-\mathrm{Mg}^{2+} /-\mathrm{Ca}^{2+}\right)$; cells were plated on coverslips coated with poly-DL-Lysine $(1 \mathrm{mg} / \mathrm{ml})$ and laminine $(20 \square / \mathrm{ml})$ and were maintained until used $\left(37^{\circ} \mathrm{C}\right.$, $\left.5 \% \mathrm{CO}_{2}\right)$, typically, for $2-3$ days.

Patch-clamp electrophysiology. Visualized patch-clamp recordings from the neutrophils were performed using a Multipatch 700B amplifier controlled by pClamp 10.2 software package (Molecular Devices, USA). For the recordings, cells on a glass coverslip were placed in a recording chamber mounted on the stage of an Olympus

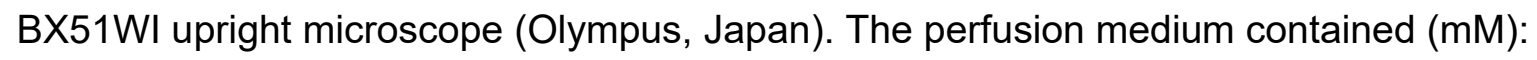
$119 \mathrm{NaCl}, 2.5 \mathrm{KCl}, 1.3 \mathrm{MgSO}_{4}, 2.5 \mathrm{CaCl}_{2}, 26.2 \mathrm{NaHCO}_{3}, 1 \mathrm{NaH}_{2} \mathrm{PO}_{4}, 10$ glucose and was continuously bubbled with $95 \% \mathrm{O}_{2}$ and $5 \% \mathrm{CO}_{2}$ (pH 7.4; 290-298 mOsm). The NMDA receptor-mediated currents were measured in voltage-clamp mode, $V_{\text {hold }}=-70$ $\mathrm{mV}$ at $33-34^{\circ} \mathrm{C}$. Signals were digitized at $10 \mathrm{kHz}$. Patch pipettes were pulled from borosilicate glass and fire-polished to 5.5-7 M $\Omega$. The intracellular pipette solution for voltage-clamp experiments contained $(\mathrm{mM}): 120.5 \mathrm{CsCl}, 10 \mathrm{KOH}-\mathrm{HEPES}, 2$ EGTA, 8 $\mathrm{NaCl}, 5 \mathrm{QX}-314 \mathrm{Br}, 2 \mathrm{Mg}-\mathrm{ATP}, 0.3 \mathrm{Na}-\mathrm{GTP}, 10 \mathrm{Na}$-phosphocreatine, pH and osmolarity adjusted to 7.2 and $295 \mathrm{mOsm}$, respectively. For whole-cell experiments in current mode, an internal solution contained (in $\mathrm{mM}$ ) $135 \mathrm{~K}$-methylsulphonate, $4 \mathrm{MgCl}_{2}$, 10 Tris-phosphocreatine, 10 HEPES, 4 MgATP, 0.4 GTP-Na (pH 7.2, osmolarity 290 $\mathrm{mOsm}$ ), the recording pipettes had the resistance of 4-7 M $\square$. Stimulating protocol consisted of a series of 500-ms depolarizing rectangular current pulses injected into a neutrophil with an increment of 20 to 30 pA (input current was injected up to $300-350$ $\mathrm{pA})$.

The fast-application system adopted by us previously (25) included a theta-glass application pipette with $\sim 200 \mu \mathrm{m}$ tip diameter attached to the PL127.11 piezo actuator driven by the E-650.00 LVPZT amplifier (both from PI Electronics). Routinely, one 
pipette channel was filled with the bath solution and the other channel was filled with the bath solution containing $100 \mu \mathrm{M}$ NMDA and $1 \mathrm{mM}$ glycine, or alternatively with NMDA, glycine plus NMDA receptors modulators. Pressure in application pipette was regulated by two-channel PDES-02DX pneumatic micro ejector (npi electronic $\mathrm{GmbH}$ ) using compressed nitrogen. To test the same using various ligands the application solutions in both theta-glass pipette channels could be exchanged within 10-12s during the experiment using dedicated pressurised micro-circuits. NMDA receptor ligands were applied in 400 ms pulses, with five-second long inter-pulse intervals.

Two-photon excitation (2PE) fluorescence imaging. For the live-cell imaging, neutrophils were loaded with a morphological tracer Cell tracker red ( $5 \mu \mathrm{M}$, Invitrogen) and $\mathrm{Ca}^{2+}$-indicator Fluo-4/AM (5 $\mu \mathrm{M}$, Invitrogen) in the presence of Pluronic F-127 $\left(0.02 \%\right.$, Invitrogen) for 10 minutes at $30^{\circ} \mathrm{C}$. After incubation with the dyes, cells were washed out for 10 minutes in a medium containing $(\mathrm{mM}): 119 \mathrm{NaCl}, 2.5 \mathrm{KCl}, 1.3$ $\mathrm{MgSO}_{4}, 2 \mathrm{CaCl}_{2}, 26 \mathrm{NaHCO}_{3}, 1.25 \mathrm{NaH}_{2} \mathrm{PO}_{4}, 12$ glucose $\left(95 \% \mathrm{O}_{2}\right.$ and $5 \% \mathrm{CO}_{2} ; \mathrm{pH}$ $7.4,290-300$ mOsm). Imaging was performed in a medium of the same composition containing either 0 or $2 \mathrm{mM} \mathrm{MgSO}_{4}$, as specified, at $30-33^{\circ} \mathrm{C}$. Imaging was carried out using an Olympus FV-1000MPE system optically linked to a Ti:Sapphire MaiTai femtosecond pulse laser (SpectraPhysics Newport) at $\lambda^{2 \mathrm{P}}{ }_{\mathrm{ex}}=800 \mathrm{~nm}$, with appropriate emission filters (Fluo-4: 515-560 nm band; Cell tracker red: 590-650 nm band), as detailed previously (Jensen et al., Elife. 2021; Henneberger et al., Neuron. 2020; Kopach et al. Biomater Sci. 2019; Scott, Rusakov. J.Neurosci. 2006). For the time-lapse recordings, z-stacks of fluorescent images (containing 5-10 cells within the field of view) were collected in a 1-min increment for 2 min before (baseline) and upon application of NMDA $(100 \mu \mathrm{M})$ and glycine $(50 \mu \mathrm{M})$ (bath application for the next $\sim 3$ minutes). At the end of each experiment, the protein kinase $C$ activator phorbol myristate acetate (PMA, $1 \mu \mathrm{M}$ ) was added for 2 min as a functional test. For the analysis, the Fluo-4 signal ( $G$, green channel) was normalised to the Cell tracker fluorescence $(R$, red channel) and changes in $\mathrm{Ca}^{2+}$ level were expressed as $\Delta G / R$ after background subtraction. Only cells displaying a stable baseline and robust (>two-fold) $\Delta G / R$ increase in response to PMA, with no Fluo-4 saturation, were included in the statistics.

Fast wide-field fluorescence imaging. Neutrophils were loaded with Fluo-4/AM (5 $\mu \mathrm{M}$, Invitrogen) in the presence of Pluronic F-127 (0.02\%, Invitrogen) for 15 minutes at $37^{\circ} \mathrm{C}$. After loading, cells were washed out for approximately 30 minutes in a Ringer solution containing (in mM) $126 \mathrm{NaCl}, 3 \mathrm{KCl}, 2 \mathrm{MgSO}_{4}, 2 \mathrm{CaCl}_{2}, 26 \mathrm{NaHCO}_{3}, 1.25 \mathrm{NaH}_{2} \mathrm{PO}_{4}, 10$ glucose, equilibrated with $95 \% \mathrm{O}_{2}$ and $5 \% \mathrm{CO}_{2}$ (pH 7.4; 290-300 mOsm). Experiments were performed at $30-33^{\circ} \mathrm{C}$, using an Olympus $\mathrm{BX} 51 \mathrm{WI}$ upright microscope (Olympus, Japan) equipped with a LUMPlanFI/IR $40 \times 0.8$ objective and an Evolve 512 EMCCD camera (Photometrics). A source of fluorescent light was an X-Cite Intelli lamp (Lumen Dynamics). For the time-lapse recordings, 700 to 3000 images were acquired in the stream-acquisition mode (exposure times from 20 to 100 ms), using MetaFluo (Cairn Research Ltd, UK) or Micromanager 4.1 (freely available ImageJ plugin) software. To collect images at high resolution, various digital zooms were used (100-200 nm per 
pixel). Changes in the intracellular $\mathrm{Ca}^{2+}$ level were expressed as the changes in Fluo-4 fluorescent signal over the baseline $\left(\square F / F_{0}\right)$ after subtraction of background fluorescence and correction for bleaching.

For mechanical stimulation, an individual cell was gently tapped with a glass pipette over the cell surface, under visual control together with monitoring changes in the pipette resistance (confirming the contact with cell membrane).

Electrochemical glutamate recordings. For the assessment of changes in the glutamate level across isolated neutrophils, we used the glutamate-sensitive electrochemical microelectrode biosensors, with a tip of 7- $\mu \mathrm{m}$ in diameter (Sarissa Biomedical Ltd., Coventry, UK). For the recordings, a glutamate biosensor was placed close to a group of neutrophils (within 5-10 $\mu \mathrm{m}$ ), with a null biosensor located apart. After positioning, the basal current was recorded for a few minutes before (baseline) and upon cell stimulation. Biosensors were calibrated prior to each experiment using 10 $\mu \mathrm{M}$ of glutamate, and the time increment was estimated.

Glutamate imaging. Fast wide-field imaging was used to visualise glutamate released by individual neutrophils in combination with an enzymatic-based assay as described earlier $(28,29)$, with some modifications. For the recordings, isolated neutrophils were perfused with a HEPES-based medium containing (in $\mathrm{mM}$ ) $135 \mathrm{NaCl}, 5 \mathrm{KCl}, 2 \mathrm{CaCl}_{2}, 2$ $\mathrm{MgCl}_{2}, 10$ HEPES, 10 glucose ( $\left.\mathrm{pH} 7.4 ; 290-300 \mathrm{mOsm}\right)$. The bath medium was supplemented with L-glutamic dehydrogenase $(\mathrm{GDH}, 60 \mathrm{U} / \mathrm{ml}$, Sigma, UK) and $\beta$ nicotinamide adenine dinucleotide (NAD, $1 \mathrm{mM}$ ). The detection of released glutamate relies on the $\mathrm{NADH}$-mediated fluorescence following the $\mathrm{GDH}$-catalyzed conversion of $\mathrm{NAD}$ to $\mathrm{NADH}$ in the presence of glutamate. Imaging was performed in a suspension of isolated neutrophils, using an Olympus BX51WI upright microscope (Olympus, Japan) equipped with a LUMPlanFI/IR $40 \times 0.8$ objective and appropriate emission filters (UMWU2 filter set, Olympus). Fluorescence was collected with a fast-speed ultrasensitive Evolve 512 EMCCD camera (Photometrics). Experiments were performed at $30-33^{\circ} \mathrm{C}$. Changes in the NADH fluorescence were calculated as $\square \mathrm{F} / \mathrm{F}_{0}$ after the subtraction of background fluorescence. Background subtraction was performed for the time-lapse sequence by subtracting the optical image before stimulation throughout $T$-stacks to eliminate basal NADH fluorescence and contaminating fluorescence, such as autofluorescence of enzymes. 


\section{References}

1. B. Amulic, C. Cazalet, G. L. Hayes, K. D. Metzler, A. Zychlinsky, Neutrophil function: from mechanisms to disease. Annu Rev Immunol 30, 459-489 (2012).

2. E. Kolaczkowska, P. Kubes, Neutrophil recruitment and function in health and inflammation. Nat Rev Immunol 13, 159-175 (2013).

3. L. G. Ng, R. Ostuni, A. Hidalgo, Heterogeneity of neutrophils. Nat Rev Immunol 19, 255-265 (2019).

4. C. Rosales, Neutrophil: A Cell with Many Roles in Inflammation or Several Cell Types? Front Physiol 9, 113 (2018).

5. M. Hager, J. B. Cowland, N. Borregaard, Neutrophil granules in health and disease. J Intern Med 268, 25-34 (2010).

6. M. Phillipson, P. Kubes, The neutrophil in vascular inflammation. Nat Med 17, 1381-1390 (2011).

7. S. L. Maas, O. Soehnlein, J. R. Viola, Organ-Specific Mechanisms of Transendothelial Neutrophil Migration in the Lung, Liver, Kidney, and Aorta. Front Immunol 9, 2739 (2018).

8. S. Nourshargh, R. Alon, Leukocyte Migration into Inflamed Tissues. Immunity 41, 694-707 (2014).

9. F. Jonsson, D. A. Mancardi, M. Albanesi, P. Bruhns, Neutrophils in local and systemic antibody-dependent inflammatory and anaphylactic reactions. J Leukoc Biol 94, 643-656 (2013).

10. F. Mollinedo, Neutrophil Degranulation, Plasticity, and Cancer Metastasis. Trends Immunol 40, 228-242 (2019).

11. B. M. Szczerba et al., Neutrophils escort circulating tumour cells to enable cell cycle progression. Nature 566, 553-557 (2019).

12. A. Z. Zucoloto, C. N. Jenne, Platelet-Neutrophil Interplay: Insights Into Neutrophil Extracellular Trap (NET)-Driven Coagulation in Infection. Front Cardiovasc Med 6, (2019).

13. C. E. M. Aarts et al., Neutrophils as Suppressors of T Cell Proliferation: Does Age Matter? Front Immunol 10, (2019).

14. S. de Oliveira, E. E. Rosowski, A. Huttenlocher, Neutrophil migration in infection and wound repair: going forward in reverse. Nature Reviews Immunology 16, 378-391 (2016).

15. J. Hann, J. L. Bueb, F. Tolle, S. Brechard, Calcium signaling and regulation of neutrophil functions: Still a long way to go. J Leukoc Biol 107, 285-297 (2020).

16. K. H. Krause, N. Demaurex, M. Jaconi, D. P. Lew, Ion channels and receptormediated Ca2+ influx in neutrophil granulocytes. Blood Cells 19, 165-173; discussion 173-165 (1993).

17. R. Immler, S. I. Simon, M. Sperandio, Calcium signalling and related ion channels in neutrophil recruitment and function. Eur J Clin Invest 48 Suppl 2 , e12964 (2018).

18. H. Jiang et al., Roles of phospholipase $\mathrm{C}$ beta2 in chemoattractant-elicited responses. Proc Natl Acad Sci U S A 94, 7971-7975 (1997).

19. D. Grimes et al., ORAI1 and ORAI2 modulate murine neutrophil calcium signaling, cellular activation, and host defense. Proc Natl Acad Sci U S A 117, 24403-24414 (2020). 
20. C. D. Collard et al., Neutrophil-derived glutamate regulates vascular endothelial barrier function. J Biol Chem 277, 14801-14811 (2002).

21. A. G. Del Arroyo et al., NMDA receptor modulation of glutamate release in activated neutrophils. EBioMedicine 47, 457-469 (2019).

22. K. T. Kotz et al., Clinical microfluidics for neutrophil genomics and proteomics. Nat Med 16, 1042-1047 (2010).

23. E. Mortaz, S. D. Alipoor, I. M. Adcock, S. Mumby, L. Koenderman, Update on Neutrophil Function in Severe Inflammation. Front Immunol 9, 2171 (2018).

24. C. L. Bashford, C. A. Pasternak, Plasma membrane potential of neutrophils generated by the Na+ pump. Biochim Biophys Acta 817, 174-180 (1985).

25. S. Sylantyev, D. A. Rusakov, Sub-millisecond ligand probing of cell receptors with multiple solution exchange. Nature Protocols 8, 1299-1306 (2013).

26. S. Dewitt, R. J. Francis, M. B. Hallett, $\mathrm{Ca}^{2+}$ and calpain control membrane expansion during the rapid cell spreading of neutrophils. J Cell Sci 126, 46274635 (2013).

27. A. V. Gourine et al., Release of ATP and glutamate in the nucleus tractus solitarii mediate pulmonary stretch receptor (Breuer-Hering) reflex pathway. J Physiol 586, 3963-3978 (2008).

28. G. S. Ayoub, S. Grutsis, H. Simko, Imaging of endogenous neurotransmitter release. J Neurosci Methods 81, 113-119 (1998).

29. P. Bezzi et al., Prostaglandins stimulate calcium-dependent glutamate release in astrocytes. Nature 391, 281-285 (1998).

30. B. Innocenti, V. Parpura, P. G. Haydon, Imaging extracellular waves of glutamate during calcium signaling in cultured astrocytes. J Neurosci 20, 1800-1808 (2000).

31. R. Gupta, D. Chattopadhyay, Glutamate is the chemotaxis-inducing factor in placental extracts. Amino Acids 37, 359-366 (2009).

32. R. Pacheco et al., Glutamate released by dendritic cells as a novel modulator of T cell activation. Journal of Immunology 177, 6695-6704 (2006).

33. Y. Ganor, M. Levite, The neurotransmitter glutamate and human T cells: glutamate receptors and glutamate-induced direct and potent effects on normal human T cells, cancerous human leukemia and lymphoma T cells, and autoimmune human T cells. J Neural Transm (Vienna) 121, 983-1006 (2014).

34. M. Schwartz, I. Shaked, J. Fisher, T. Mizrahi, H. Schori, Protective autoimmunity against the enemy within: fighting glutamate toxicity. Trends Neurosci 26, 297302 (2003).

35. A. A. Boldyrev, D. O. Carpenter, P. Johnson, Emerging evidence for a similar role of glutamate receptors in the nervous and immune systems. J Neurochem 95, 913-918 (2005).

36. T. W. Hodo, M. T. P. de Aquino, A. Shimamoto, A. Shanker, Critical Neurotransmitters in the Neuroimmune Network. Front Immunol 11, 1869 (2020).

37. M. A. Park, M. J. Lee, S. H. Lee, D. K. Jung, J. Y. Kwak, Anti-apoptotic role of phospholipase $\mathrm{D}$ in spontaneous and delayed apoptosis of human neutrophils. FEBS Lett 519, 45-49 (2002). 